substantially to important metabolic complications, such as type 2 diabetes and fatty liver. Given the urgent need for more effective therapies for these common diseases, it will be essential to identify molecular mediators of increased endocannabinoid activity in obesity. As with many complex diseases, it is likely that endocannabinoid dysregulation in obesity will arise at the intersection between genetic susceptibility (e.g., polymorphisms in endocannabinoid pathway genes) and an obesogenic metabolic environment, including overnutrition, suboptimal dietary lipid composition, inactivity, insulin/leptin resistance, and tissue inflammation. Studies are required to both fully dissect these possibilities and to identify optimal dietary and lifestyle interventions useful to attenuate endocannabinoid overactivity (20). Parallel identification of additional pharmacologic compounds that can reduce activity of the cannabinoid system is clearly needed. However, the critical challenge will be to verify the absence of CNS effects of endocannabinoid modulation in longer-term studies in both preclinical models and humans, so that we are not doomed to rehash the unfortunate saga of rimonabant.

\section{Acknowledgments}

The author gratefully acknowledges research support from NIH grant DK062948 (to M.E. Patti), the American
Diabetes Association, the Lilly Foundation, and the Graetz Fund.

Address correspondence to: Mary-Elizabeth Patti, Joslin Diabetes Center, Boston, Massachusetts 02215, USA. Phone: 617.735.1966; Fax: 617.732.2593; E-mail: mary.elizabeth.patti@joslin.harvard.edu.

1. Gaoni Y, Mechoulam R. Isolation, structure, and partial synthesis of an active constituent of hashish. J Am Chem Soc. 1964;86(8):1646-1647.

2. Matsuda LA, Lolait SJ, Brownstein MJ, Young AC, Bonner TI. Structure of a cannabinoid receptor and functional expression of the cloned cDNA. Nature. 1990;346(6284):561-564.

3. Munro S, Thomas KL, Abu-Shaar M. Molecular characterization of a peripheral receptor for cannabinoids. Nature. 1993;365(6441):61-65.

4. Premkumar LS, Ahern GP. Induction of vanilloid receptor channel activity by protein kinase C. Nature. 2000;408(6815):985-990.

5. Lauckner JE, Jense JB, Chen H-C, Lu H-C, Hille B, Mackie K. GPR 55 is a cannabinoid receptor that increases intracellular calcium and inhibits $M$ current. Proc Natl Acad Sci U S A. 2008;105(7):2699-2704.

6. Di Marzo V. Targeting the endocannabinoid system: to enhance or reduce? Nat Rev Drug Discov. 2008;7(5):438-455.

7. Lambert DM, Muccioli GG. Endocannabinoids and related $\mathrm{N}$-acylethanolamines in the control of appetite and energy metabolism: emergence of new molecular players. Curr Opin Clin Nutr Metab Care. 2007;10(6):735-744.

8. Scheen AJ, Finer N, Hollander P, Jensen MD, Van Gaal LF. Efficacy and tolerability of rimonabant in overweight or obese patients with type 2 diabetes: a randomised controlled study. Lancet. 2006;368(9548):1660-1672.

9. Christensen R, Kristensen PK, Bartels EM, Bliddal $\mathrm{H}$, Astrup A. Efficacy and safety of the weight-loss drug rimonabant: a meta-analysis of randomised trials. Lancet. 2007;370(9600):1706-1713.

10. Osei-Hyiaman D, et al. Hepatic CB1 receptor is required for development of diet-induced steatosis, dyslipidemia, and insulin and leptin resistance in mice. J Clin Invest. 2008;118(9):3160-3169.

11. Bluher $M$, et al. Dysregulation of the peripheral and adipose tissue endocannabinoid system in human abdominal obesity. Diabetes. 2006;55(11):3053-3060.

12. Motaghedi R, McGraw TE. The CB1 endocannabinoid system modulates adipocyte insulin sensitivity. Obesity (Silver Spring). 2008;16(8):1727-1734.

13. Cavuoto P, McAinch AJ, Hatzinikolas G, CameronSmith D, Wittert GA. Effects of cannabinoid receptors on skeletal muscle oxidative pathways. Mol Cell Endocrinol. 2007;267(1-2):63-69.

14. Artmann A, et al. Influence of dietary fatty acids on endocannabinoid and $\mathrm{N}$-acylethanolamine levels in rat brain, liver and small intestine. Biochim Biophys Acta. 2008;1781(4):200-212.

15. Bermudez-Silva FJ, Suarez PJ, Nadal A, Rodriguez de Fonseca F. The role of the pancreatic endocannabinoid system in glucose metabolism. Best Pract Res Clin Endocrinol Metab. 2009;23(1):87-102.

16. Engeli S, et al. Activation of the peripheral endocannabinoid system in human obesity. Diabetes. 2005;54(10):2838-2843.

17. Ravinet TC, et al. Anti-obesity effect of SR141716, a CB1 receptor antagonist, in diet-induced obese mice. Am J Physiol Regul Integr Comp Physiol. 2003; 284(2):R345-R353

18. Ravinet TC, Delgorge C, Menet C, Arnone M, Soubrie P. CB1 cannabinoid receptor knockout in mice leads to leanness, resistance to diet-induced obesity and enhanced leptin sensitivity. Int J Obes Relat Metab Disord. 2004;28(4):640-648.

19. Tam J, et al. Peripheral CB1 cannabinoid receptor blockade improves cardiometabolic risk in mouse models of obesity. J Clin Invest. 2010; 120(8):2953-2966.

20. Di Marzo V, et al. Changes in plasma endocannabinoid levels in viscerally obese men following a 1 year lifestyle modification programme and waist circumference reduction: associations with changes in metabolic risk factors. Diabetologia. 2009;52(2):213-217.

\title{
Mice that "conditionally" lack basophils, AT LAST
}

\section{Booki Min}

\author{
Department of Immunology, Lerner Research Institute, Cleveland Clinic Foundation, Cleveland, Ohio, USA.
}

\begin{abstract}
Basophils are the least abundant granulocytes found in the circulation. Until recently, their functions were poorly understood. In the past few years, the list of basophil functions in the context of immunity has dramatically increased. Thus, the need for basophil-deficient animal models to confirm these findings is imperative. In this issue of the JCI, Wada and colleagues introduce the first mouse model in which basophils are conditionally ablated in vivo. Using this model, they then uncover a nonredundant role for basophils in acquired immunity against tick infection.
\end{abstract}

Basophils, the least abundant granulocytes found in the circulation, have long been out-

Conflict of interest: The author has declared that no conflict of interest exists.

Citation for this article: J Clin Invest. 2010; 120(8):2648-2651. doi:10.1172/JCI44058. siders in the immunology community. This is in part because of their scarcity and phenotypic similarity to mast cells. Although mast cells are not classified as granulocytes, they share several characteristics with basophils; these include surface expression of the high-affinity $\mathrm{Fc}$ receptor for $\operatorname{IgE}$ ( $\mathrm{Fc \varepsilon RI}$ ) and the ability to release chemical mediators such as histamine after stimulation. In mice, mature basophils have a relatively small cytoplasm and few cytoplasmic granules (1). However, surface markers that are specific for basophils have not been identified; thus, an experimental tool to examine the unique functions of basophils in vivo has long been wanted. Recent studies have increased the need for this, as they have accumulated convincing evidence that basophils play crucial roles in the development of immune responses, particularly those associated with type 2 immunity $(2,3)$.

Basophil-mediated regulation of immune responses is primarily medi- 
A Primary infestation

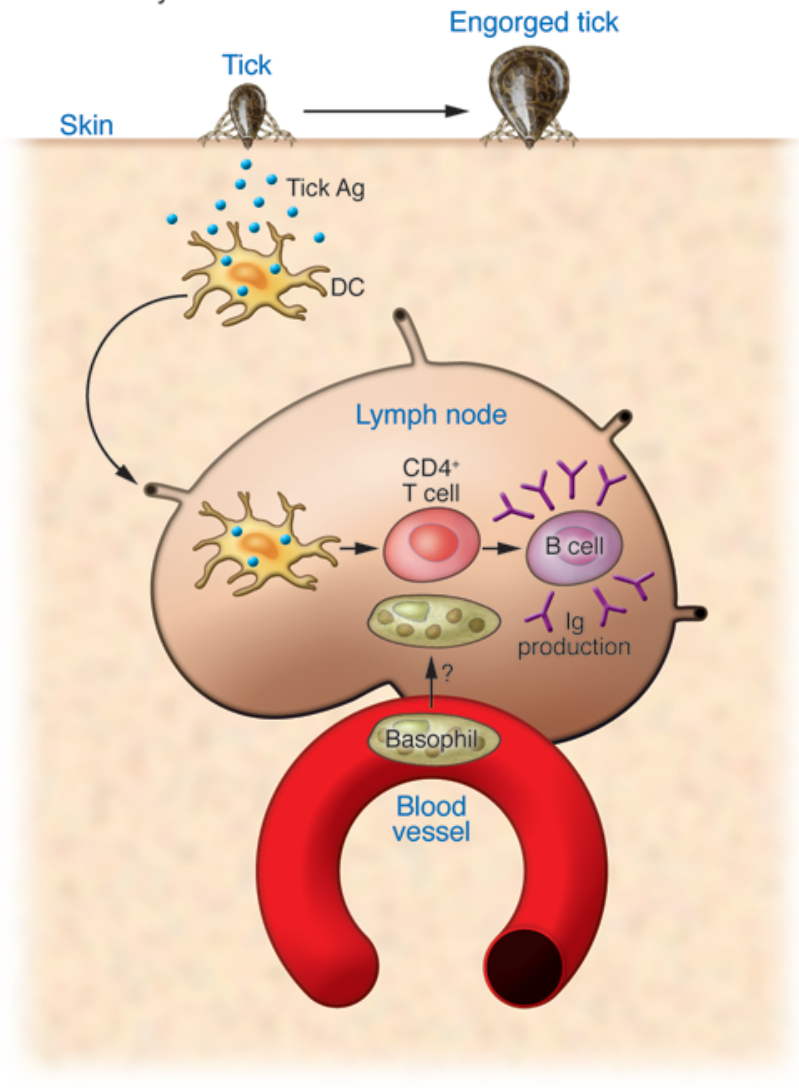

B Secondary infestation

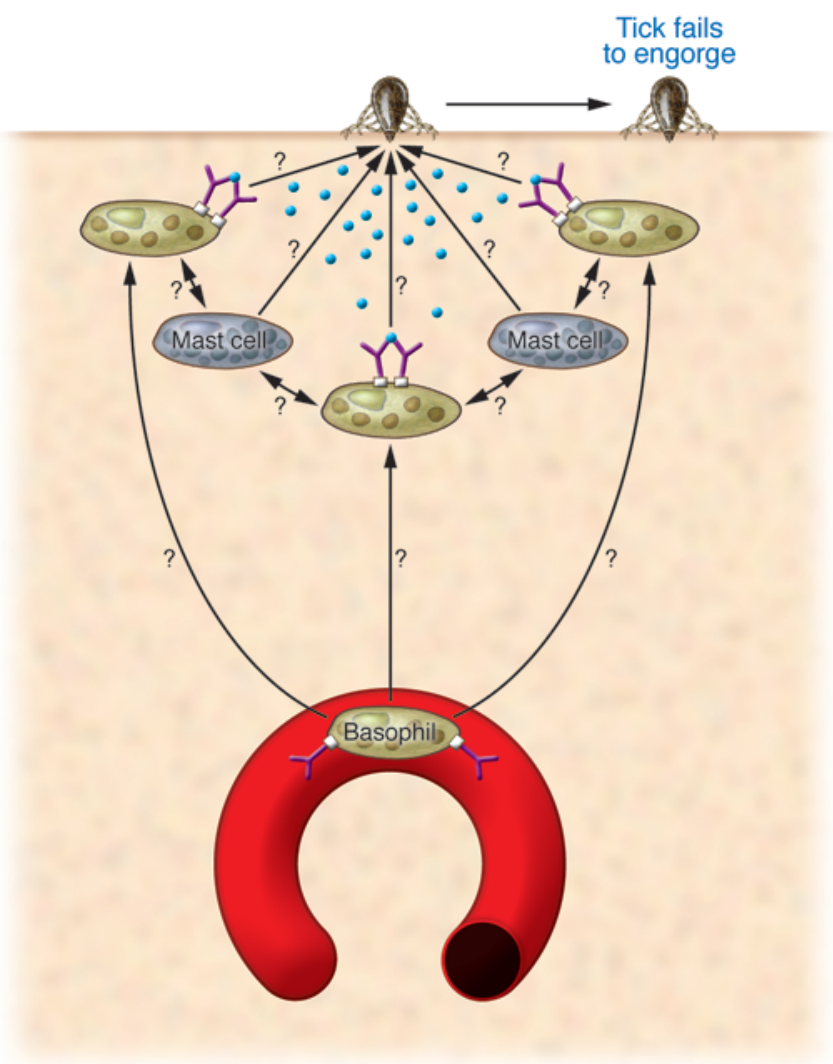

\section{Figure 1}

Basophil responses during primary and secondary tick infestation. The model depicted here for the role of basophils during primary and secondary tick infestation as suggested by the data of Wada and colleagues (12). (A) Primary infestation. Upon infestation, tick antigens are likely to be taken up by tissue-resident dendritic cells, which subsequently migrate to the draining lymph nodes, where they present the antigen to CD4+ T cells. Activated CD4+ T cells are expected to provide "help" to B cells to produce tick antigen-specific Igs (including IgE and IgG). Whether basophils enter into the draining lymph nodes and influence the T cell responses as previously seen in the $N$. brasiliensis infection model remains unclear. Tick engorgement takes place. (B) Secondary infestation. Upon secondary infestation, basophils are rapidly recruited to the infestation sites, where they are expected to be activated by surface antibody crosslinking. How basophil recruitment is induced is unknown. Tissue mast cells play an important role in providing resistance, although antibody-mediated activation of mast cells is dispensable for the resistance. Antibody-activated basophils may cooperate with mast cells during this process. Tick engorgement fails to occur. The effector mechanisms that directly inhibit tick engorgement remain to be examined.

ated by the Th2-type cytokines produced by basophils, including IL-4 and thymic stromal lymphopoietin. Unlike Th2 CD4 ${ }^{+}$ $\mathrm{T}$ cells, which arise following stimulation of naive $\mathrm{CD}^{+} \mathrm{T}$ cells in the appropriate cytokine milieu, basophils do not require a differentiation step to produce Th2-type cytokines; instead, it has been shown that they spontaneously acquire the capacity to produce these cytokines (4). Importantly, basophils have been shown to enter antigen-draining lymph nodes where they support Th2 differentiation of naive $\mathrm{CD}^{+}$ T cells (5). Furthermore, basophils have also been demonstrated to function as antigenpresenting cells that directly prime antigen-specific naive $\mathrm{CD}^{+} \mathrm{T}$ cells to become Th2 effector $\mathrm{T}$ cells even in the absence of CD $11 c^{+}$dendritic cells (6). Despite this increasing evidence that basophils regulate type 2 immunity, their contribution to protective effector immunity remains less well characterized. A substantial increase in the number of IL-4-producing basophils was found in mice infected with the intestinal nematode Nippostrongylus brasiliensis (7), although their role as inducers of $N$. brasiliensis-specific Th2 immunity was recently challenged (8). Basophils also have recently been found in the lamina propria of the small intestine and the lung parenchyma during infection of mice with $N$. brasiliensis (9), and depletion of basophils using Ab treatment impaired parasite expulsion during primary and secondary infection with $N$. brasiliensis $(9,10)$, suggesting that baso- phils may play a protective role against the parasite infection.

\section{Basophil-deficient mice}

As the number of functions ascribed to basophils has risen, so the demand for an animal that lacks basophils has continued to rise. In vivo depletion using monoclonal Abs specific for FceRI (MAR-1) or CD200R3 (Ba103) has at least allowed researchers to begin to test the functions of basophils in vivo $(5,11)$. However, concerns related to the possibility that basophils are activated by the $A b$ treatment during depletion, which would result in cytokine secretion, and/or the possibility that the Abs cross-react with nonbasophils, particularly mast cells, have always been an 
issue of uncertainty. In this issue of the JCI, Wada et al. report for the first time a mouse model of inducible basophil ablation (12). The mouse model was generated by introducing the diphtheria toxin receptor-encoding (DTR-encoding) gene into the $3^{\prime}$ untranslated region of the mast cell serine protease 8 (Mcpt8) gene in a bacterial artificial chromosome. Consistent with a report that mouse MCP8 is a basophilspecific differentiation marker (13), expression of DTR was only found in basophils and not in other cells, especially mast cells. Treatment of the mice, which were termed $M c p t 8^{D T R}$ mice, with diphtheria toxin (DT) led to a transient depletion of basophils in the bone marrow and periphery, while the majority of mast cells remained unaffected during the treatment.

\section{Tick infection model}

Studies using guinea pigs and ruminants have shown that repeated tick infestation induces cutaneous basophilia and that host Fc receptors are required for Ab-mediated resistance $(14,15)$. Moreover, the recruited basophils appear to play an important role in host resistance, as injection of anti-basophil serum partially impairs resistance to infestation (16). However, this notion was challenged by the finding that mast celldeficient mice fail to acquire resistance to tick infestation (17) and by the difficulty in identifying infiltrating basophils in tissues stained with $\mathrm{H} \& \mathrm{E}$.

However, using their mouse model of inducible basophil ablation, Wada et al. have provided indisputable evidence that it is indeed basophils that have a nonredundant role in Ab-mediated acquired immunity against reinfestation with the tick Haemaphysalis longicornis, which is the vector that mediates babesiosis, Q fever, and Russian encephalitis in humans (12). The authors established a model system in which mice infested with $H$. longicornis ticks acquire protective immunity during primary infestation, as determined by substantially reduced numbers and weights of engorged ticks (a measure known as tick repletion) during the second infestation compared with the first. Consistent with an earlier report (17), tick repletion remained unaltered in mice deficient in mast cells $\left(K i t^{W-s h / W-s h}\right)$ and diminished to the level seen in wild-type mice upon reconstituting $K i t^{W-s b / W-s h}$ mice with mast cells derived from wild-type bone marrow, suggesting that mast cells play a key role in the acquisition of resistance to tick reinfestation. Moreover, both antibodydeficient and Fcer1g-deficient mice failed to display tick resistance, supporting the idea that anti-tick antibodies (probably IgE isotype) mediate resistance. However, Kit ${ }^{\mathrm{W}-}$ $s h / W-s h$ mice that received mast cells derived from Fcer $1 \mathrm{~g}$-deficient bone marrow were fully capable of lowering tick repletion, as seen in recipients of mast cells derived from wild-type bone marrow, suggesting that Ig receptors on mast cells are not necessary for Ab-mediated resistance to tick reinfestation. This unexpected observation prompted the authors to test the role of basophils in this experimental setting.

Wada et al. then used the $M c p t 8^{D T R}$ mice to directly test the role of basophils in acquired resistance to tick infection (12). Treatment of $M c p t 8^{D T R}$ mice with DT during the secondary infestation completely abolished resistance. Importantly, the total mast cell numbers in the tick feeding sites remained unchanged relative to control mice, strongly indicating that mast cells are not necessary for resistance. The importance of basophils was further supported by the fact that transfer of basophil-enriched CD $49 \mathrm{~b}^{+}$ splenocytes isolated from sensitized mice was sufficient to confer resistance in naive mice. Moreover, transferring CD49b ${ }^{+}$splenocytes isolated from DT-treated (but not from PBS-treated) $M c p t 8^{D T R}$ mice that had been infested once was unable to diminish tick repletion. These results strongly suggest that basophils play a critical nonredundant role in acquired resistance to tick reinfestation. The authors further showed that the transfer of CD $49 \mathrm{~b}^{+}$splenocytes isolated from tick-infested Fcer $1 \mathrm{~g}$-deficient mice failed to confer resistance; in other words, the expression of Ig receptors on basophils rather than mast cells is a key determinant for protection, confirming their earlier finding of resistance following reconstitution of $K i t^{W-s h / W-s h}$ mice with mast cells derived from Fcer1g-deficient bone marrow.

\section{Future perspectives}

The work of Wada et al. (12) raises several important questions for future study. A cellular mechanism underlying basophil recruitment to sites of tick infestation needs to be examined (Figure 1). Basophils are found within the draining lymph nodes after primary infection with $N$. brasiliensis, and IL-3 is necessary for this recruitment (8). It is therefore possible that circulating basophils may enter lymph nodes that drain tick antigens during primary infestation and that those basophils then present tick antigens and induce type 2 differentiation. The significance of basophil recruitment to the infestation sites during secondary infection is more likely related to their effector function. While the source of IL-3 in this setting is likely activated $\mathrm{CD} 4^{+} \mathrm{T}$ cells, the target cells of IL- 3 are less well characterized. Because IL- 3 is believed to induce the production of chemokines and expression of adhesion molecules that contribute to basophil transendothelial migration (18), finding the IL-3-responding target cells is likely to illuminate the mechanism involved in basophil migration. However, basophil migration into lymphoid versus nonlymphoid parenchymal tissues including skin or lung might utilize different mechanisms.

How recruited basophils mediate resistance also needs to be characterized. Given the failure of Fcer1g-deficient splenocytes to restore tick resistance in $M c p t 8^{D T R}$ mice treated with DT, it is likely that tick-specific antibodies crosslink Ig receptors on basophils that then secrete mediators that deter tick engorgement. However, whether basophils are directly involved in anti-tick immunity or recruit other effectors such as eosinophils into the sites (16) to mediate resistance remains to be explored. It is important to note that despite the fact that mast cells are necessary for acquired resistance to tick infestation, a mechanism mediating the resistance appears to be distinct from that mediated by basophils. What is particularly striking is that failure of resistance in mast cell-deficient mice is reversed by the transfer of mast cells derived from Fcer $1 \mathrm{~g}$ deficient bone marrow. Given the fact that depletion of basophils alone is sufficient to abolish resistance, it is likely that mast cells are needed for basophils to execute anti-tick (Ig-dependent) effector immunity. Understanding the cellular cooperation between mast cells and basophils will be an important subject of investigation.

In sum, the results reported by Wada et al. (12) identify a previously unrecognized effector role for basophils during infestation with the tick H. longicornis, an important vector mediating critical human diseases. Moreover, the availability of an animal in which basophils can be conditionally deleted will allow us to better understand the mystery of these rare cells in the immune system.

\section{Acknowledgments}

The author is supported by NIH grant AI080908. 
Address correspondence to: Booki Min, Department of Immunology/NB30, Lerner Research Institute, Cleveland Clinic Foundation, Cleveland, Ohio 44195, USA. Phone: 216.445.3126; Fax: 216.444.8372; E-mail: minb@ccf.org.

1. Dvorak AM, Nabel G, Pyne K, Cantor H, Dvorak $\mathrm{HF}$, Galli SJ. Ultrastructural identification of the mouse basophil. Blood. 1982;59(6):1279-1285.

2. Min B. Basophils: what they 'can do' versus what they 'actually do'. Nat Immunol. 2008;9(12):1333-1339.

3. Karasuyama H, Mukai K, Tsujimura Y, Obata K. Newly discovered roles for basophils: a neglected minority gains new respect. Nat Rev Immunol. 2009;9(1):9-13.

4. Gessner A, Mohrs K, Mohrs M. Mast cells, basophils, and eosinophils acquire constitutive IL-4 and IL-13 transcripts during lineage differentiation that are sufficient for rapid cytokine production. J Immunol. 2005;174(2):1063-1072.

5. Sokol CL, Barton GM, Farr AG, Medzhitov R. A mechanism for the initiation of allergeninduced T helper type 2 responses. Nat Immunol. 2008;9(3):310-318.
6. Siracusa MC, Perrigoue JG, Comeau MR, Artis D. New paradigms in basophil development, regulation and function. Immunol Cell Biol. 2010; 88(3):275-284

7. Min B, et al. Basophils produce IL-4 and accumulate in tissues after infection with a Th2-inducing parasite. J Exp Med. 2004;200(4):507-517.

8. Kim S, Prout M, Ramshaw H, Lopez AF, LeGros G, Min B. Cutting edge: basophils are transiently recruited into the draining lymph nodes during helminth infection via IL-3, but infection-induced Th2 immunity can develop without basophil lymph node recruitment or IL-3. J Immunol. 2010;184(3):1143-1147.

9. Ohnmacht C, Voehringer D. Basophil effector function and homeostasis during helminth infection. Blood. 2009;113(12):2816-2825.

10. Ohnmacht C, Voehringer D. Basophils protect against reinfection with hookworms independently of mast cells and memory Th2 cells. J Immunol. 2010;184(1):344-350

11. Obata $\mathrm{K}$, et al. Basophils are essential initiators of a novel type of chronic allergic inflammation. Blood. 2007;110(3):913-920

12. Wada T, et al. Selective ablation of basophils in mice reveals their nonredundant role in acquired immunity against ticks. J Clin Invest. 2010;
120(8):2867-2875.

13. Poorafshar M, Helmby H, Troye-Blomberg $M$, Hellman L. MMCP-8, the first lineage-specific differentiation marker for mouse basophils. Elevated numbers of potent IL-4-producing and MMCP-8positive cells in spleen of malaria-infected mice. Eur I Immunol. 2000;30(9):2660-2668.

14. Brown SJ, Askenase PW. Amblyomma americanum: requirement for host $F_{C}$ receptors in antibodymediated acquired immune resistance to ticks. Exp Parasitol. 1985;59(2):248-256.

15. Monteiro GE, Bechara GH. Cutaneous basophilia in the resistance of goats to Amblyomma cajennense nymphs after repeated infestations. Ann NY Acad Sci. 2008;1149:221-225.

16. Brown SJ, Galli SJ, Gleich GJ, Askenase PW. Ablation of immunity to Amblyomma americanum by anti-basophil serum: cooperation between basophils and eosinophils in expression of immunity to ectoparasites (ticks) in guinea pigs. J Immunol. 1982;129(2):790-796.

17. Matsuda H, Fukui K, Kiso Y, Kitamura Y. Inability of genetically mast cell-deficient W/Wv mice to acquire resistance against larval Haemaphysalis longicornis ticks. J Parasitol. 1985;71(4):443-448.

18. Iikura $M$, et al. Transendothelial migration of human basophils. J Immunol. 2004;173(8):5189-5195.

\title{
Seeing red: flushing out instigators of niacin-associated skin toxicity
}

\author{
Richard L. Dunbar 1,2,3,4 and Joel M. Gelfand $2,5,6$
}

\begin{abstract}
${ }^{1}$ Department of Medicine, Division of Experimental Therapeutics, ${ }^{2}$ nstitute for Translational Medicine and Therapeutics, ${ }^{3}$ Institute for Diabetes, Obesity, and Metabolism, ${ }^{4}$ The Cardiovascular Institute, ${ }^{5}$ Department of Dermatology, Department of Epidemiology, and Department of Biostatistics, and ${ }^{6}$ Center for Clinical Epidemiology and Biostatistics, University of Pennsylvania, Philadelphia, Pennsylvania, USA.
\end{abstract}

The use of niacin to improve plasma lipid levels and reduce risk of myocardial infarction is limited by noxious skin effects that result from stimulation of G protein-coupled receptor 109A (GPR109A) in skin immune cells. Niacin causes vasodilation, manifest as rubor (redness) of the head and neck, providing a visible sign associated with other, more bothersome skin complaints. The working theory is that niacin provokes Langerhans cells to produce prostaglandin $\mathrm{D}_{2}\left(\mathrm{PGD}_{2}\right)$, stimulating vascular DP1 receptors to cause vasodilation. In this issue of the JCI, Hanson and colleagues raise a serious challenge to this paradigm in showing that the major player in vasodilation is the keratinocyte, which produces $\mathrm{PGE}_{2}$, stimulating $\mathrm{EP}_{2 / 4}$ receptors, shifting the role of the Langerhans $/ \mathrm{PGD}_{2} / \mathrm{DP}_{1}$ pathway to that of an accomplice. They also show that the antipsoriasis drug monomethyl fumarate, itself a GPR109A agonist, provokes vasodilation through the same cells. These efforts bring us one step closer to solving a key limitation of an important cardioprotective drug and reveal that the skin response to niacin is much more complicated than previously thought.

The therapeutic use of niacin has a long and colorful history (1). Remarkably, it has played a critical role in

Conflict of interest: R.L. Dunbar declares conflicts with Abbott and Merck and Company. J.M. Gelfand declares conflicts with Amgen, Abbott, Centocor, Pfizer, Novartis, Merck and Company, and Celgene.

Citation for this article: J Clin Invest. 2010; 120(8):2651-2655. doi:10.1172/JCI44098. preventing a top cause of death in 2 different centuries by 2 seemingly unrelated mechanisms. It eradicated pellagra, a vitamin deficiency disease caused by a chronic lack of niacin (vitamin $\mathrm{B}_{3}$ ) as a source of $\mathrm{NAD}^{+}$. Before niacin was added to the food supply, pellagra was a major cause of morbidity and mortality in the early 20th century. Presumably through its beneficial effects on lipid levels, niacin also prevents myocardial infarction (MI), a public health menace in the early 21st century (2). Statins have justifiably replaced niacin as the first-line therapy for the treatment of hyperlipidemia; however, even statins only reduce coronary heart disease (CHD) risk by about one-quarter, leaving considerable residual risk in vulnerable populations. Thus, niacin is reemerging as an adjunct to statin therapy to reduce the incidence of CHD events even further. Niacin is also recommended as an adjunct to statin therapy to raise HDL cholesterol and lower triglycerides (2). Although niacin has been used for more than 50 years to lower cholesterol levels, we are only now starting to piece together the molecular mechanism by which it does this, following the discovery that $\mathrm{G}$ protein-coupled receptor 109A (GPR109A) is a receptor for niacin that mediates some of its myriad effects (3-5). GPR109A is highly expressed in adipocytes, where stimulation results in the profoundly antilipolytic effect of niacin and, in turn, the acute reduction in levels of plasma free 